\title{
Probabilistic Design of Distribution \& Reticulation Systems juxtaposed with Traditional Design Software
}

\author{
E. Chikuni ${ }^{1}$, S. Arendese ${ }^{2}$, O.I. Okoro ${ }^{3}$ C.C. Awah $^{3}$, O Dzobo ${ }^{4}$ \\ ${ }^{1}$ Department of Electrical Engineering, University of Zimbabwe, \\ ${ }^{2}$ City of Cape Town, South Africa \\ ${ }^{3}$ Department of Electrical and Electronics Engineering, Michael Okpara University of Agriculture, \\ Umudike, Nigeria \\ ${ }^{4}$ Department of Electrical Engineering, University of Johannesburg
}

echikuni@ieee.org, shannon.arendse@yahoo.com,odzobo@yahoo.com, oiokoro@yahoo.co.uk, ccawah@ieee.org

\begin{abstract}
A residential development design using the Herman Beta method based on a widely used software package ReticMaster for low voltage and DIgSILENT for medium voltage (which is also used for more complex analysis and higher voltage design). The design was done to meet the requirement according to National Energy Regulator of South Africa (NERSA) standards. Cost comparisons were carried to determine materials best suited for the design. The Herman-Beta method is a departure from other probabilistic methods, in particular those based on Gaussian assumption. It applies to the South African distribution systems which have less customers per distribution point, typically less than 30 [2].
\end{abstract}

Keywords: Low voltage; medium voltage design; Herman-Beta method; distribution system and residential development design, system reliability.

\section{Introduction}

The power system which supplies electricity to the consumer consists of a generation, transmission and distribution system. In this paper only the distribution system will be discussed. The distribution system is divided into a medium voltage (e.g. $11 \mathrm{kV})$ and a low voltage (eg. 400V). A distribution system consists of feeders, distributors and service mains. In the past the radial system was used, but to ensure continuity of supply the ring-main distributor system is used. Distribution substations' main function is to step-down the voltage to the required level. Low voltage distribution planning involves the placement of miniature substations at strategic areas to provide optimal supply to consumers. The low voltage distribution system is the final stage in ensuring quality of supply to the consumer [1]. According to the South African National Rationalization of Standards document NRS034-1, the voltage drop for low voltage cables should not be higher than 10\% [2]. Therefore the voltage drop is the main constraint which determines the size of cable required [2]. According to the NRS guidelines [5] voltage drop limits must comply with the following maximum limits:

MV distributor $\quad 3 \%$

LV feeder $\quad 8 \%$

ACRID 2017, June 20-21, Victoria Falls, Zimbabwe

Copyright $(2017$

DOI 10.4108/eai.20-6-2017.2271003 
Historically the deterministic method was used to calculate the voltage drop in low voltage cables [3]. The disadvantage of this method was the correction factor applied to correct diversity and unbalance in electrification systems became uncertain and assumptions were made [3]. Assumptions could lead to over- or under- designs which results in unnecessary expenditure or poor quality of supply [4].

The study to determine what type of load domestic consumers started in 1987, where data loggers were development in South Africa [5]. A research study was done in 1994 in which various sites in different parts of the country, 60 or more sampled households were monitored individually on site using data loggers recording the average current per 5 minute sample rate [5]. A survey was also conducted per household which was used to categorised different consumer load class [2], [6]. For analysis the load data collected where presented in a histogram [5]. The study revealed that the classification of loads into low, high, medium and the assumption of a Gaussian (normal) distribution was simplistic and could be costly.

The Herman Beta method designed by Ron Herman uses the Beta probability density function (pdf), with a risk level or certainty to determine the voltage drop of a cable. Beta parameter plots have been made for many districts in South Africa and result from recording load currents over 5-minute periods. Longer periods can be extrapolated from the 5 minute data, but not the other way round.

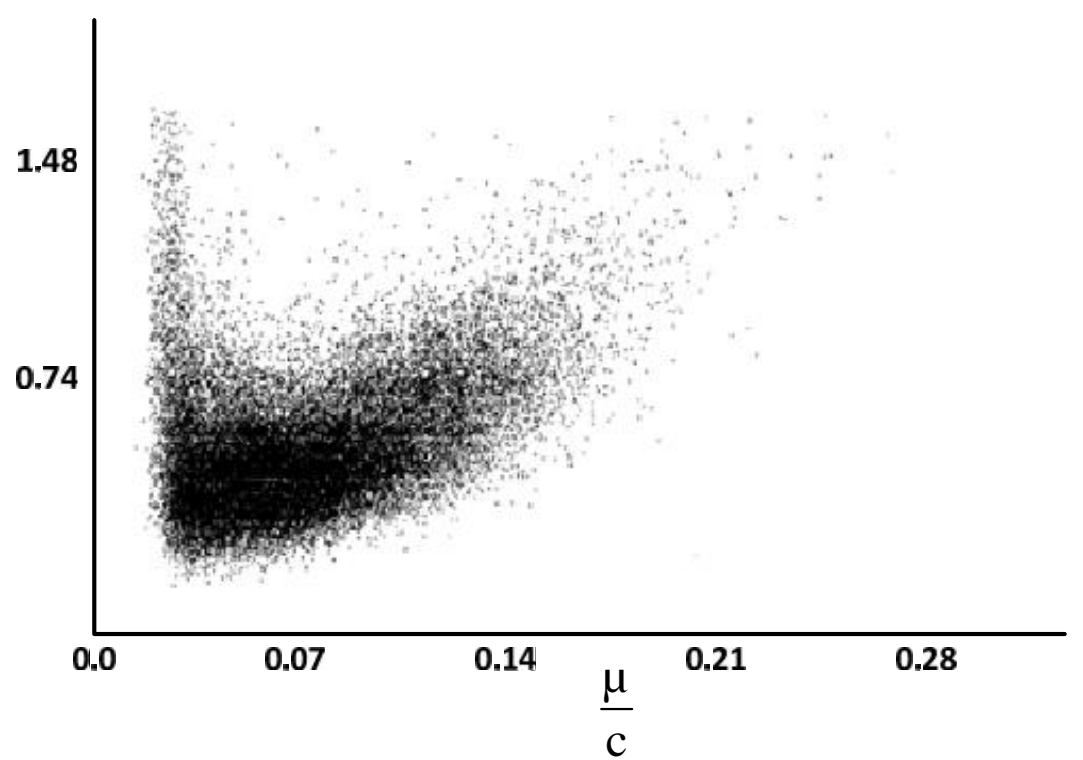

Fig.1 Beta parameter for Helderberg, South Africa (July-December, 1997 

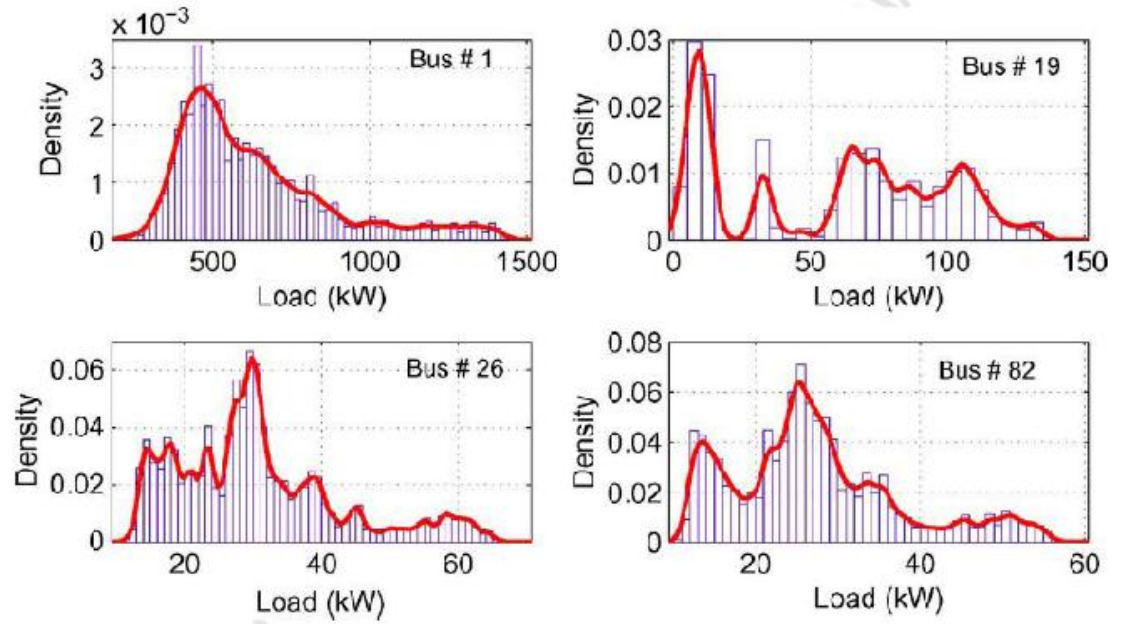

Figure 2, Probability distribution of load at different buses (Sing et al)

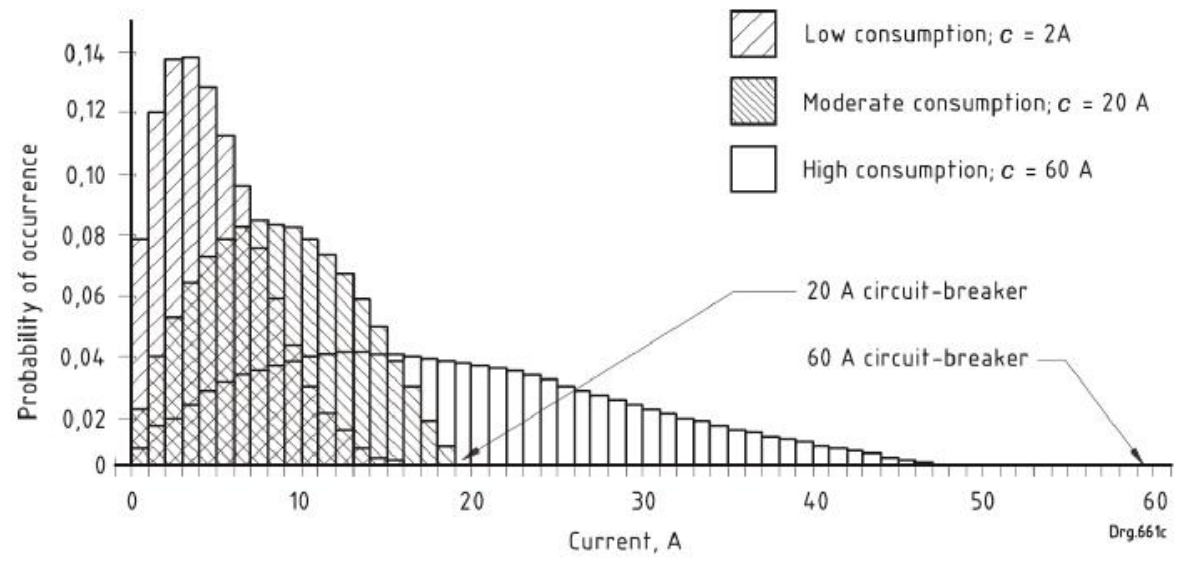

Figure 3, Typical Load Distributions

Thousands of measurements are made and the resulting effective parameters / variables to define the Beta pdf are $\mu, \sigma, \alpha, \beta$ and $\mathrm{c}$.

Where, $\mu=$ customer demand, or after diversity maximum demand, $\sigma=$ standard deviation, $\beta$, the Beta parameter, and $\mathrm{c}$ the scaling factor (circuit breaker rating). The relation between the variables is: 


$$
\begin{aligned}
& \alpha=-\left(s^{2}-1\right) \frac{\mu}{c}+s^{2} \\
& \frac{\alpha}{\beta}=\frac{\frac{\mu}{c}}{1-\frac{\mu}{c}}
\end{aligned}
$$

\begin{tabular}{|c|c|c|c|c|c|c|c|c|c|c|c|c|c|c|c|c|}
\hline \multirow{3}{*}{ Current type } & 2 & 3 & 4 & 5 & & 6 & 7 & 8 & & 9 & 10 & 11 & 12 & \begin{tabular}{|r|}
13 \\
\end{tabular} & 14 & 15 \\
\hline & & & & & & \multicolumn{4}{|c|}{ Load parameters -7 years } & & & & & \begin{tabular}{|l|} 
Load \\
parameter \\
s - 15 \\
years
\end{tabular} & & \\
\hline & & Income rangeb & & & & & ADMD & & $A$ & $\sigma A$ & & & & ADMD & $A$ & $\sigma A$ \\
\hline Consumer & AMPSa and & (gross & $a$ & $b$ & & c & kVA & & & & $a$ & $b$ & c & kVA & & \\
\hline class & LSMa class & R/month) & & & & & & & & & & & & & & \\
\hline Rural & LSM 1 & 0 to 600 & 0,30 & 2,98 & & 20 & 0,42 & 1,83 & & 2,78 & 0,35 & 2,88 & 20 & 0,50 & 2,17 & 3,03 \\
\hline settlement & (low end) & & & & & & & & & & & & & & & \\
\hline Rural village & LSM 1 and 2 & 400 to 900 & 0,43 & 2,52 & & 20 & 0,67 & 2,91 & & 3,55 & 0,48 & 2,13 & 20 & 0,84 & 3,65 & 4,07 \\
\hline Informal & LSM 3 and 4 & 800 to 1500 & 0,77 & 9,88 & 60 & & 1,00 & & 4,35 & 4,56 & 0,91 & 8,80 & 60 & 1,30 & 5,56 & 5,36 \\
\hline \multicolumn{17}{|l|}{ settlement } \\
\hline Tow nship area & LSM 5 and 6 & 1500 to 3000 & 1,05 & 7,81 & 60 & & 1,64 & & 7,13 & 6,18 & 1,22 & 5,86 & 60 & 2,37 & 10,30 & 7,96 \\
\hline Urban & LSM7 & 3000 to 5500 & 1,23 & 5,56 & 60 & & 2,50 & & 10,87 & 8,28 & 1,25 & 3,55 & 60 & 3,59 & 15,61 & 10,93 \\
\hline \multicolumn{17}{|l|}{ residential I } \\
\hline Urban & LSM 7 and 8 & 5500 to 8500 & 1,45 & 6,07 & 80 & & 3,54 & & 15,39 & 10,81 & 1,42 & 4,10 & 80 & 4,72 & 20,52 & 13,68 \\
\hline \multicolumn{17}{|l|}{ residential II } \\
\hline Urban tow nship & LSM 8 & 8500 to 12000 & 1,45 & 5,75 & & 80 & 3,70 & 16,09 & & 11,20 & 1,42 & 4,13 & 80 & 4,70 & 20,43 & 13,63 \\
\hline \multicolumn{17}{|l|}{ complex } \\
\hline Urban multi- & LSM 8 (high & 12000 to 24000 & 1,43 & 4,41 & & 80 & 4,50 & 19,57 & & 13,15 & 1,37 & 3,39 & 80 & 5,30 & 23,04 & 15,09 \\
\hline storey/estate f & end) & & & & & & & & & & & & & & & \\
\hline
\end{tabular}

\subsection{Departure from Traditional Voltage Drop Methods}

The HB method as applied to South African domestic electricity load regime simplifies the model of a feeder to be just resistive and still getting reliable results, while avoiding complex modelling (Figure 2).
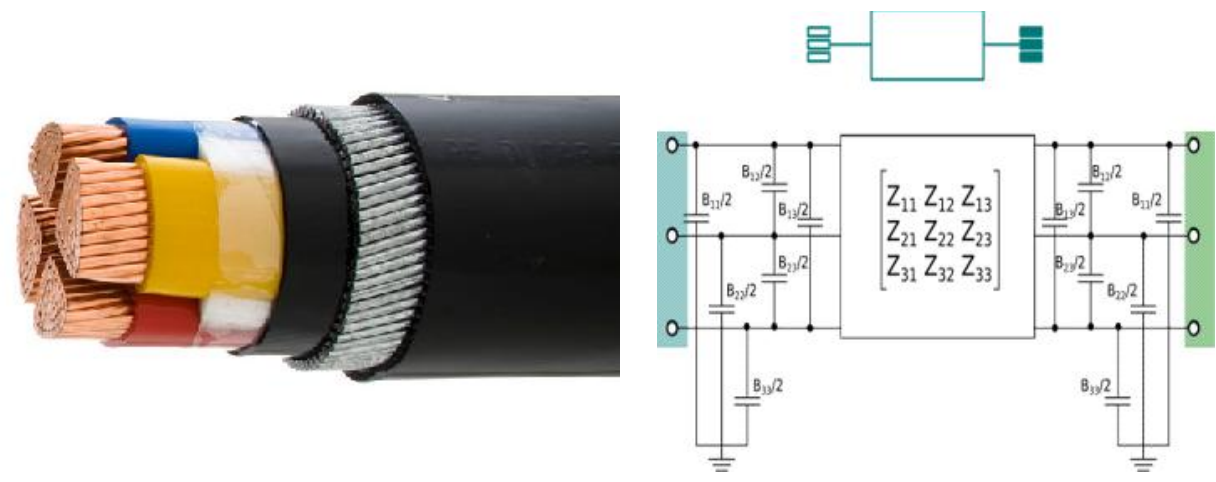

Figure 2: Three phase cable and its generalised representation (Berkeley Lab) 


\subsection{Example}

An example to illustrate the concepts will be in the design of an electrical distribution network to supply a new urban residential development. The Herman Beta method is used to determine the size of the low voltage cables. Two software packages are used for load flow studies, DIgSILENT for medium voltage (MV) and ReticMaster for low voltage (LV) network. The results are verified by hand calculations and a NRS034 spreadsheet adapted for "Local Authority A" usage. The design is done to meet the quality of supply and performance requirements in accordance to the National Energy Regulator of South Africa (NERSA) standards.

\section{Methodology}

\subsection{Preliminary Data Gathering}

The preliminary phase of the design required a certain amount of information to be availed by the utility, in this case a municipal undertaking, specifically a plans for the new residential development. Information on the number of consumers and the general disposition was also required. Such information would have a direct bearing on the supply side MVA. From the information on the location miniature substations and kiosks were placed. The lengths of feeder cables were also determined. Figure 3, illustrates the process. Table 1 is the customer supplied data.

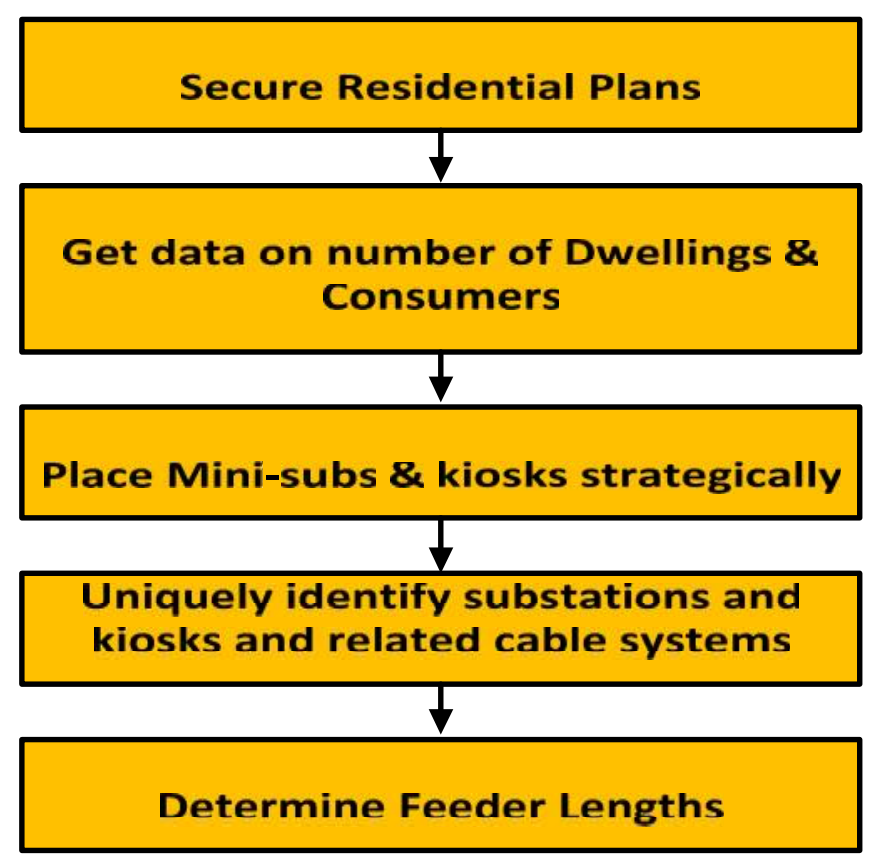


Fig.3 Information and Data CollectionTable 1, Customer Supplied Data

Table 1, Customer Supplied Data

\begin{tabular}{llll}
\hline LAND USE & $\begin{array}{c}\text { NO OF } \\
\text { UNITS }\end{array}$ & $\begin{array}{l}\text { ELECTRICAL } \\
\text { ALLOWANCE }\end{array}$ & $\begin{array}{l}\text { ELECTRICAL } \\
\text { DEMAND }\end{array}$ \\
\hline Single residential & 363 & $5.31 \mathrm{kVA} / \mathrm{unit}$ & $1927.53 \mathrm{kVA}$ \\
& & & \\
Group housing & 123 & $4.04 \mathrm{kVA} / \mathrm{unit}$ & $496.92 \mathrm{kVA}$ \\
Duplex & 94 & $4.04 \mathrm{kVA} / \mathrm{unit}$ & $379.76 \mathrm{kVA}$ \\
Flats & 223 & $4.04 \mathrm{kVA} / \mathrm{unit}$ & $900.92 \mathrm{kVA}$ \\
Primary school & 1 & $70 \mathrm{kVA} / \mathrm{unit}$ & $70 \mathrm{kVA}$ \\
Place of worship & 1 & $25 \mathrm{kVA} / \mathrm{unit}$ & $25 \mathrm{kVA}$ \\
Creche & 1 & $25 \mathrm{kVA} / \mathrm{unit}$ & $25 \mathrm{kVA}$ \\
Total load & $\mathbf{8 0 6}$ & & $\mathbf{3 8 2 5} \mathbf{k V A}$ \\
\hline
\end{tabular}

\subsection{Modelling Stage, ReticMaster $@$}

\subsubsection{Settings}

Low voltage modelling was done with a software package ReticMaster ${ }^{\odot}$, which is widely in the industry in South Africa. The following calculation method settings were invoked:

$\rightarrow$ Calculation Method: Statistical Herman Beta.

$\Rightarrow$ Application: Domestic.

$\rightarrow$ Statistical Parameters: Single phase $\alpha=1.05 ; \beta=1.70$ and $\operatorname{Imax}=60$.

$\rightarrow$ Confidence: $90 \%$

$\rightarrow$ Select Beta Currents

\subsubsection{Design}

$\rightarrow$ Select the relevant voltage conductor type and cable size; length of cable; amount of domestic connections per kiosk or bulk supply kVA, and confirm that the voltage drop along each low voltage feeder cable does not exceed the design limit of $8 \%$.

$\rightarrow$ Conduct a cost comparison to determine the ideal conductor between copper and aluminium. 


\section{Medium Voltage Side Stud}

DIgSILENT / Power Factory was used for the Medium Voltage / 11kV side of the network with the following data input.

\begin{tabular}{lll}
\hline \multicolumn{1}{c}{ Grid } & Transformer & \multicolumn{1}{c}{ General Load } \\
\hline External Grid: & Two-winding & Input mode: \\
PV bus type & Transformer & S, cos $\varphi$ \\
Bus bar: & Rated Power & (Apparent power \\
Rated voltage & HV side Voltage & Power factor) \\
Rated current & LV side Voltage & \\
Source Impedance & Vector Group & \\
S/C Limit: 21 kA & Per Unit Impedance & \\
S/C Duration 1 sec & Tap changer details & \\
& & \\
\hline
\end{tabular}

Load flow was carried out after inputting the relevant data.

\section{Results}

Table 2: Cable Size Selected, Initial Results

\begin{tabular}{|c|c|c|c|}
\hline FROM & TO & CABLE SIZE & $\%$ VOLTAGE \\
\hline B3 & 1B3 & $95 \mathrm{~mm}^{2} \mathrm{AL}$ & $96.88 \%$ \\
\hline $1 \mathrm{~B} 3$ & $2 \mathrm{~B} 3$ & $70 \mathrm{~mm}^{2} \mathrm{AL}$ & $92.65 \%$ \\
\hline $2 \mathrm{~B} 3$ & 3B3 & $25 \mathrm{~mm}^{2} \mathrm{AL}$ & $86.28 \%$ \\
\hline B3 & $4 \mathrm{~B} 3$ & $95 \mathrm{~mm}^{2} \mathrm{AL}$ & $96.62 \%$ \\
\hline $4 \mathrm{~B} 3$ & $5 \mathrm{~B} 3$ & $35 \mathrm{~mm}^{2} \mathrm{AL}$ & $86.09 \%$ \\
\hline B3 & $6 \mathrm{~B} 3$ & $35 \mathrm{~mm}^{2} \mathrm{AL}$ & $91.78 \%$ \\
\hline B3 & 7B3 & $70 \mathrm{~mm}^{2} \mathrm{AL}$ & $95.97 \%$ \\
\hline 7B3 & $8 B 3$ & $25 \mathrm{~mm}^{2} \mathrm{AL}$ & $91.67 \%$ \\
\hline
\end{tabular}

In Table 2, there are four instances in which the $8 \%$ maximum voltage drop threshold was violated. In two instances the differences from the $8 \%$ datum were of the order of $0.3 \%$, and therefore no change of cable section was deemed necessary. The next available higher cable section was used for the non-compliant cables. The affected cables are given in Table 3. 
Table 3: Changed Cables

\begin{tabular}{|l|l|l|l|}
\hline FROM & TO & CABLE SIZE & \% VOLTAGE \\
\hline $2 \mathrm{~B} 3$ & $3 \mathrm{~B} 3$ & $70 \mathrm{~mm}^{2} \mathrm{AL}$ & $92.21 \%$ \\
\hline $4 \mathrm{~B} 3$ & $5 \mathrm{~B} 3$ & $70 \mathrm{~mm}^{2} \mathrm{AL}$ & $92.02 \%$ \\
\hline
\end{tabular}

\section{Discussion and Conclusions}

In this paper we have investigated the probabilistic design method in concept as well as from the viewpoints of other practitioners internationally. We have noted that it is not always good practice to use standard packages and apply them without assessing their suitability as well as the cost and reliability implications of the results. The HB method has been adopted in South Africa, but caution must be exercised in adopting software that already suggests data for South Africa, as such data may not apply for other regions, even around Southern Africa. Thus there will be a need for individual countries to collect their own data, which as we have seen can take several years. We have found that even applying the HB method with Retic Master, which with $\mathrm{HB}$ options, in the end we must use Engineering judgement to come up final design parameters.

\section{References}

Dr E. Chikuni, Dr O.I. Okoro \& Prof M.T. Khan. Electrical Engineering. Cape Town, South Africa: Juta \& Company Ltd, 2008, pp. 405.

[2] I. Ferguson \& C.T. Gaunt."LV network sizing in electrification projects - replacing a deterministic method with a probabilistic method," presented at the 17th Int. Conf. Electricity Distribution, Barcelona, 2003.

[3] R. Herman \& C.T. Gaunt, A Modern approach to Reticulation design, Univ. of Stellenbosch, short course 120, ver. $2.1,1997$.

[4] M. Dekenah.”NRS034 Domestic load research project 1994-2009.” Pretoria, South Africa

[5] NRS, Rationalized User Specification, Guidelines for the provision of Electrical Distribution networks in residential areas, part 1: Planning and design of distribution systems, NRS 034-1: 1997.

[6] City of Cape Town Technical Standards, Electrical reticulation, CTEF 100: 2005

[7] “Grid Electricity," in Guidelines for human settlement planning and design, Ch $12.1 \mathrm{pp} .1$

[8] H.C. Kadada,'Designing Low Voltage Feeders to meet Quality of Supply specifications for voltage variations". H.C. Kadada, MSc Thesis, University of Cape Town

[9] C T Gaunt, R Herman, M Dekenah, R L Sellick, S W Heunis, "Data collection, load modelling and probabilistic analysis for lv domestic electrification”, CIRED, 1999

[10] J. Dickert, P. Schegner, "Residential Load Models for Network PlanningPurposes", Proceedings of the International Symposiumn on Modern Electric Power Systems (MEPS), 2010. 